\title{
Optical properties of in-vitro biomineralised silica
}

SUBJECT AREAS: BIOTECHNOLOGY

OPTICAL MATERIALS AND DEVICES

MATERIALS CHEMISTRY

BIOPHOTONICS

Received

12 March 2012

Accepted

9 August 2012

Published

29 August 2012

\section{Correspondence and} requests for materials should be addressed to A.P. (apolini@lbl.gov) or D.P. (dario. pisignano@unisalento.

* Current address:

Lawrence Berkeley National Laboratory,

Materials Sciences

Division, 1 Cyclotron

Road, 94720

Berkeley, CA, USA.

\# Current address: Cavendish Laboratory, University of Cambridge, J. J. Thomson Avenue, Cambridge CB3 OHE, United Kingdom.

\author{
Alessandro Polini ${ }^{1 *}$, Stefano Pagliara ${ }^{1 \#}$, Andrea Camposeo ${ }^{1,2}$, Roberto Cingolani ${ }^{3}$, Xiaohong Wang ${ }^{4}$, \\ Heinz C. Schröder ${ }^{4}$, Werner E. G. Müller ${ }^{4}$ \& Dario Pisignano ${ }^{1,2,5}$
}

\begin{abstract}
${ }^{1}$ National Nanotechnology Laboratory of Istituto Nanoscienze-CNR, Università del Salento, via Arnesano, 1-73100 Lecce, Italy, ${ }^{2}$ Center for Biomolecular Nanotechnologies@UNILE, Istituto Italiano di Tecnologia, Via Barsanti, 1-73010 Arnesano-LE, Italy, ${ }^{3}$ Italian Institute of Technology, via Morego 30, 1-16163 Genova, Italy, ${ }^{4}$ Institute for Physiological Chemistry, University Medical Center of the Johannes Gutenberg University, Duesbergweg 6, D-55128 Mainz, Germany, ${ }^{5}$ Dipartimento di Matematica e Fisica "Ennio De Giorgi", Università del Salento, via Arnesano, 1-73100 Lecce, Italy.
\end{abstract}

Silicon is the second most common element on the Earth's crust and its oxide $\left(\mathrm{SiO}_{2}\right)$ the most abundant mineral. Silica and silicates are widely used in medicine and industry as well as in micro- and nano-optics and electronics. However, the fabrication of glass fibres and components requires high temperature and non-physiological conditions, in contrast to biosilica structures in animals and plants. Here, we show for the first time the use of recombinant silicatein- $\alpha$, the most abundant subunit of sponge proteins catalyzing biosilicification reactions, to direct the formation of optical waveguides in-vitro through soft microlithography. The artificial biosilica fibres mimic the natural sponge spicules, exhibiting refractive index values suitable for confinement of light within waveguides, with optical losses in the range of 5-10 $\mathrm{cm}^{-1}$, suitable for application in lab-on-chips systems. This method extends biosilicification to the controlled fabrication of optical components by physiological processing conditions, hardly addressed by conventional technologies.

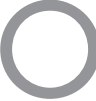

rganisms in nature are able to control the composition, crystallography, morphology, and material properties of organic-inorganic composites and biominerals in a highly ordered way at nanoscale, by biomineralisation processes ${ }^{1,2}$. While some species populate extreme geothermal or hydrothermal, strongly acidic environments, or tolerate hundreds of $\mathrm{atm}^{3}$, most of them live under mild chemical, $\mathrm{pH}$, and pressure conditions. Controlling biomineralisation reactions would allow to carry out the syntheses of inorganic materials in-vitro, possibly near room temperature, in aqueous solution, and at or near neutral $\mathrm{pH}$, namely in a "green" way alternative to traditional materials-processing techniques. Furthermore, the exploitation of polymers, proteins and peptides for biomineralisation may enable a fine control on size, shape, and structure of the inorganic products ${ }^{4-8}$, important for their incorporation in devices ${ }^{9}$.

Among the most important materials in industry, silica is daily used in the production of glasses, ceramics, catalysts, as a food additive, as well as an insulator in semiconductor electronics and optical medium in photonic crystals $^{10-12}$. The industrial production and processing of silica typically needs caustic chemicals and high temperatures $\left(400-2300^{\circ} \mathrm{C}\right)^{13}$, at extreme values of pressure (i.e. down to below $10^{-2} \mathrm{~atm}$ in various types of chemical vapour deposition reactors ${ }^{14}$ and $\mathrm{pH}^{15}$. On the contrary, several single- and multicellular organisms are able to realize species-specific 2D and 3D nano- and microstructures in-vivo under physiological conditions ${ }^{1}$. In particular, biosilica of the siliceous sponges (phylum Porifera) forms the spicules, intricate and complex skeletal elements that support and protect the organisms, at a purity reaching that of quartz glass ${ }^{16}$. In fact, sponge spicules can work as waveguides for light, relying on total internal reflection to confine photons in either a single-mode or a multi-mode optical signal ${ }^{17-20}$. Transmission losses, roughly estimated in the range of $10^{-4}-10^{-1} \mathrm{~cm}^{-118,20}$, are still higher than those in commercial fibres (by about four orders of magnitude), but compatible with values required for short distance data communication or on-chip microscale waveguides ${ }^{21,22}$. In particular, the siliceous sponges have the unique ability to synthesize biosilica enzymatically, using a specific enzyme, termed silicatein ${ }^{23}$, which is a member of the cathepsin proteinase family ${ }^{24-28}$. In the present work, we use recombinant silicatein (from the demosponge Suberites domuncula) for the control of the biomineralisation process, and direct the formation of biosilica microstructures in-vitro through biocompatible, soft lithography. The optical properties of the in-vitro obtained biosilica are assessed, and light propagation losses at optical wavelength measured for the first time, highlighting the analogies between our biosilicificated components and naturally-formed, microscopic spicules. The patterning method extends biosilicification to the controlled fabrication of microstructures by 
a

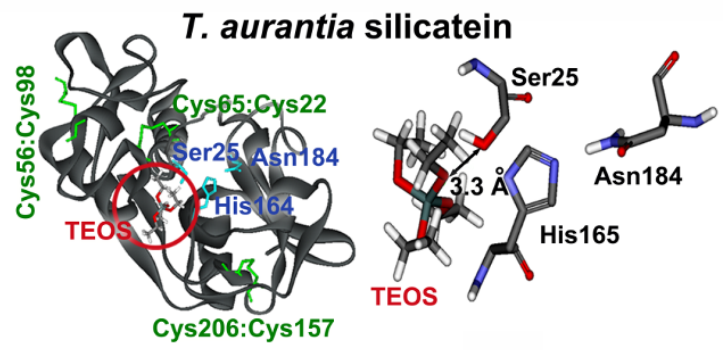

b

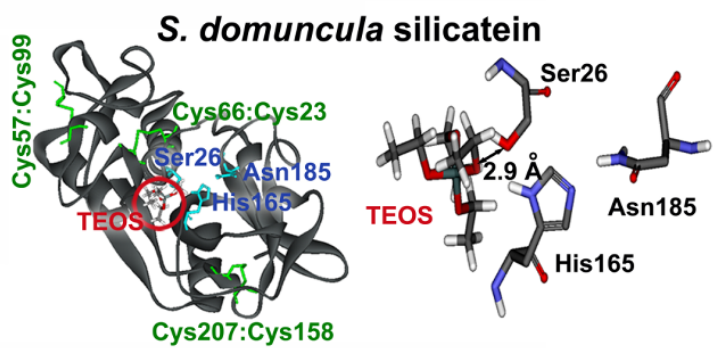

C

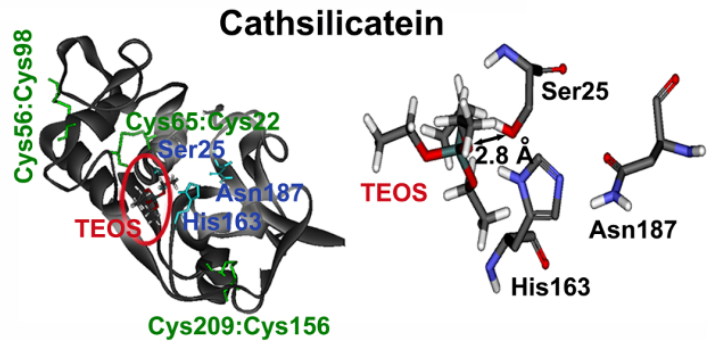

Figure $1 \mid$ Models of silicatein. (a-c) Models of silicatein- $\alpha$ from $T$. aurantia (a) and $S$. domuncula (b) based on the crystal structure of cathepsin $\mathrm{S}^{29}$. The structure for cathsilicatein is also given (c). The amino acids of the catalytic triad, in silicatein [Ser, His, and Asn], are highlighted together with the three disulfide bridges. The most likely position for the insertion of TEOS into the active centre is circled (in red). Right: sterical proximity of the three amino acids, comprising the active centre, to the TEOS molecule. The distances between Ser hydroxyl group and TEOS are indicated.

physiological processing conditions, which, thought useful for applications of optical detection and sensing in lab on chips, cannot be addressed by conventional technologies.

\section{Results}

Comparing the Tethya aurantia and the $S$. domuncula silicatein- $\alpha$, and the cathsilicatein of known crystal structure, a model of silicatein- $\alpha$ has been predicted using the most related human cathepsin $S$ as a template (SWISSPROT approach). While the amino acids of the catalytic triad are located centrally within the molecule (Fig. 1, left), the Ser-rich residues of the hydroxyl amino acid cluster are located at the protein surface. The active centres of the proteins allow the docking of a monomeric silica precursor such as tetraethoxysilane (TEOS), with an optimal distance to the hydroxyl group of Ser computed to be below $3.3 \AA$, thus favouring hydrolytic reactions (Fig. 1 , right; see also Supplementary Information) and the nanoscale control of biosilification.

For the realization of biosilica microstructures, here the recombinant protein is first patterned by microfluidics on a hydroxylated $\mathrm{Si} / \mathrm{SiO}_{2}$ substrate, exploiting an elastomeric mold and external pumping at a flux of $500 \mu \mathrm{L} / \mathrm{h}$, and then incubated in a TEOS solution for $120 \mathrm{~h}$ after peeling off (Fig. 2a). Fig. 2b displays the self-assembly of patterned recombinant silicatein. The protein layer mostly exhibits linear, parallel-oriented protruding features (highlighted by rectangles in Fig. 2b), which indicate assembled filaments aligning along the flow direction. From binarised SEM images (Fig. 2c), we calculate a value of $\approx 1.7$ for the fractal dimension, $D$, useful for describing the degree of complexity of the microfabricated structure and especially relevant to the path of filament formation and to the biological functionality of silicatein ${ }^{30} .100 \mu \mathrm{m}$-wide biomineralised fibres, long up to $1 \mathrm{~cm}$, are obtained in two different types: as adhered microstructures on Si substrates ( 90\% of $100 \mu \mathrm{m}$-high, $100 \mu \mathrm{m}$ wide and $10 \mathrm{~mm}$-long features present on the elastomeric mould), and as free-standing wires ( $\sim 10 \%$; inset of Fig. $2 \mathrm{~d})$, that accidentally remain entrapped in form of protein fibres into the mould recessed features during the mechanical peeling-off of PDMS. The latter are then released upon immersion of the mould in TEOS solution, since PDMS partially loses its conformability and sticking properties. This entrapment phenomenon is likely related to local disuniformities of the $\mathrm{PDMS} / \mathrm{SiO}_{2}$ conformal contact during pressure-driven fluidics (see the full Materials and Methods section in the Supplementary Information for further details). Both before and after TEOS incubation, major protein constituents $(\mathrm{C}, \mathrm{N}$, and $\mathrm{O})$ are clearly present due to the persistent presence of silicatein in the inner shell of the microstructures, whereas after mineralization a remarkable increase is found in the oxygen content, together with a significant presence of silicon (Fig. 2d). Control experiments, applying this method in the absence of proteins or with non-enzymatically working proteins such as collagen ${ }^{31}$, evidence ineffective biomineralisation (see Supplementary Information), thus highlighting the role of silicatein.

To assess the optical properties of the obtained material, we investigate the refractive index $(n)$, by confocal imaging light backreflected from the interfaces between borosilicate glass and recombinant silicatein and biosilica, respectively. In fact, $n$ values of the compounds constituting the microstructure are crucial for the photon waveguiding. The optical discontinuity between two dielectric media (e.g. borosilicate/protein and borosilicate/biosilica, respectively) critically affects the reflection of photons ${ }^{32}$. Consequently, confocal mapping of light reflected from interfaces allows to measure the refractive index of a material, once the $n$ value of the facing compound is known (for details see Supplementary Information). Using incident light with wavelength, $\lambda$, of $405 \mathrm{~nm}$ by a diode laser, we map the refractive index of the surface of 100 $\mu \mathrm{m}$-wide deposited silicatein and of biosilica features (Fig. 3a). The same approach is applied to a cleaved microstructure, exploiting the reflection at the air/biosilica interface, to achieve a cross-sectional map of refractive index (Fig. 3b). A measurement of the dependence of the refractive index of biosilica on the light wavelength is performed by an Ar laser as photon source $(\lambda=457.9,488.0$ and $514.5 \mathrm{~nm}$, Table 1). The as-obtained $n$ data are described by a Cauchy model ${ }^{32}, n(\lambda)=A+B \lambda^{-2}$, where $A$ and $B$ are materialdependent constants. The optical transmission properties of the fibres are characterized by a laser beam $(\lambda=633 \mathrm{~nm})$. The coupling of the laser light is optimized by maximizing the optical power transmitted by both free-standing fibres and fibres deposited on fused quartz. The light is collected through an objective lens with low numerical aperture (N.A. $=0.10$ ), and measured with a charge coupled device (CCD) camera. We find that the light transmitted by the fibres and collected in a plane $(x, y)$ perpendicular to the waveguide axis $(z)$ is in an almost circular spot representing the radiation modes propagating into the biosilica waveguide and then coupling to free space (Fig. 3e). The intensity profiles along $y$ and $x$ are roughly fitted by a Gaussian function (continuous curves in Fig. 3d and 3e, respectively).

By measuring the waveguide divergence, namely the half width at half maximum (HWHM) angle of transmitted light, as a function to the coupling angle at the entrance of the fibres ${ }^{33}$, an acceptance angle of about $20^{\circ}$ (full acceptance angle $40^{\circ}$ ) is found for the biosilica fibres. The power of the transmitted light $\left(P_{\text {out }}\right)$ is related to the power coupled into the line $\left(P_{i n}\right)$ by an exponential decay law, 
a

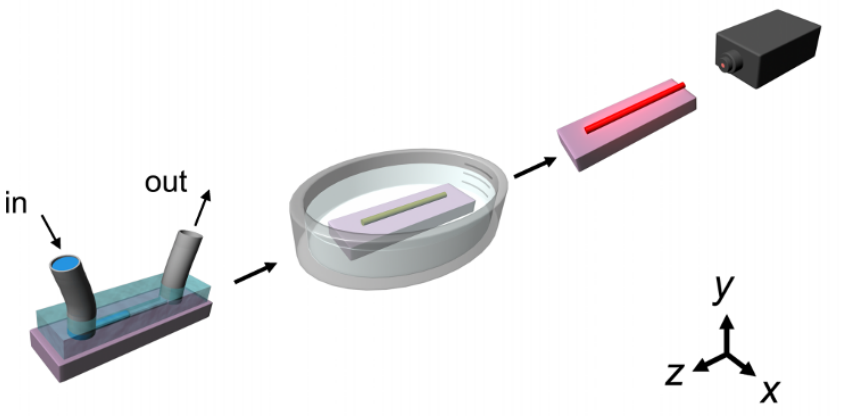

b

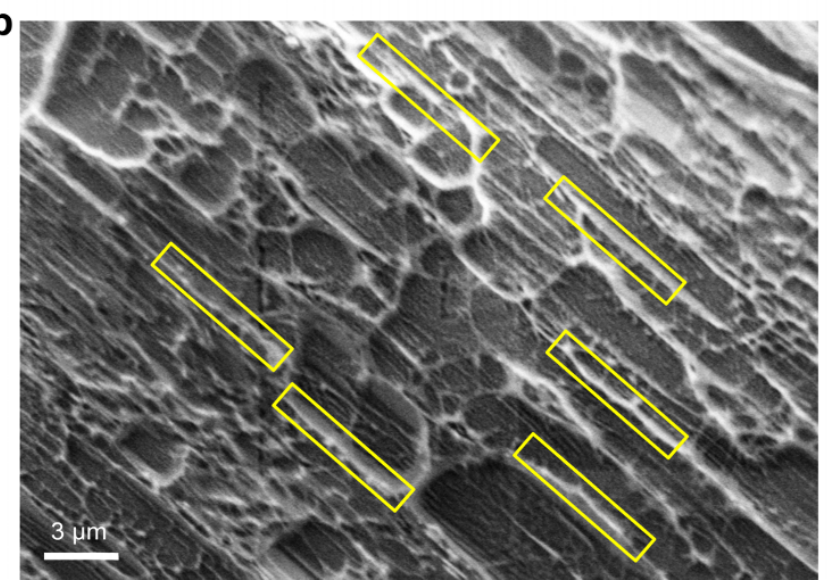

C

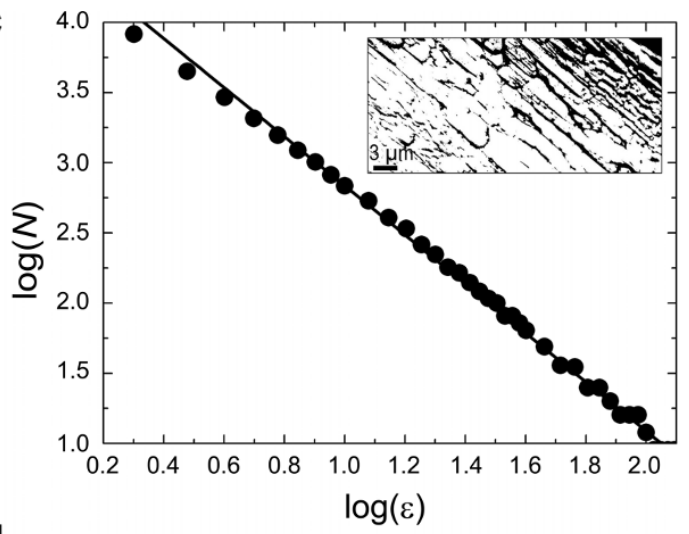

d

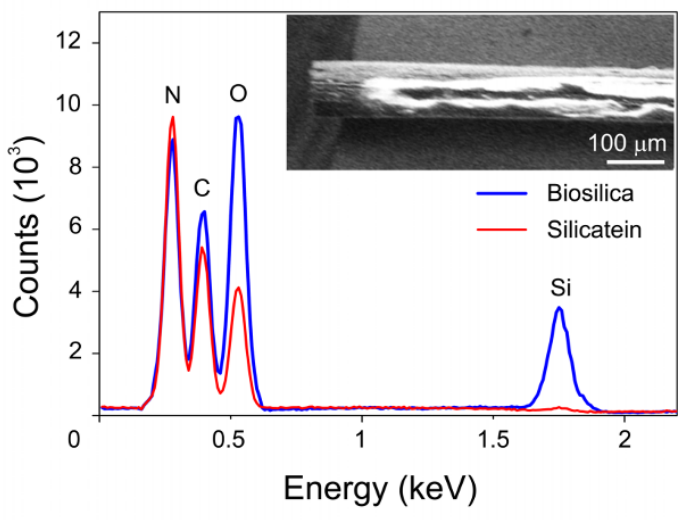

Figure 2 | Fabrication steps, structural and elemental characterization of lithographically-made biomineralised waveguides by recombinant silicatein$\boldsymbol{\alpha}$. (a) Silicatein patterning using pressure-driven microfluidic flow, within a device assembled by a poly(dimethylsiloxane) (PDMS) elastomeric element and a Si substrate. Small black arrows indicate the microflow direction. After peeling off the elastomer, incubation in a TEOS solution results in biosilicification directed by the patterned protein. Finally, the waveguiding properties of coupled laser light by the biomineralised microstructures are investigated. The system of coordinates $(x, y, z)$ used to identify the longitudinal axis of the realized fibres is also shown in the lower right corner.

(b) Scanning electron microscopy (SEM) micrograph of the patterned silicatein surface evidencing the presence of almost parallel, linear protruding microscale features, highlighted by rectangles. (c) Log-log box-counting plot, showing the number of pixels $(N)$ per box (falling in the image), against the box size, $\varepsilon$, with superimposed linear fitting line. The fit yields the value, $D=(1.75 \pm 0.01)$. Inset: Corresponding binary image of the region of interest, obtained by SEM of patterned recombinant silicatein. (d) Structural and morphological characterization: energy dispersive $\mathrm{x}$-ray spectroscopy (EDS) spectra of patterned features, before (i.e. silicateins, red line) and after (i.e. mainly biosilica, blue line) biosilicification. Inset: SEM micrograph of a biosilica fibre deposited on fused quartz.

$P_{\text {out }}=P_{\text {in }} e^{-\alpha L}$, where $\alpha$ is the propagation loss coefficient and $L$ indicates the waveguide length. This expression is valid for uniformly absorbing or scattering waveguiding media. We measure the propagation loss coefficient of photons travelling in the microstructure, by measuring $P_{\text {in }}$ and $P_{\text {out }}$ for the laser beam coupled into the biosilica waveguides (about ten samples with known length). The $\alpha$ values are also estimated by measuring the intensity $(I)$ of the light diffused from the surface of the fibre body, as a function of the distance, $d$, from the face of entrance of input light. Fig. 4a shows a typical plot of the log of $I$ against the plot of $d$ for free-standing wires, yielding a straight line whose slope corresponds to the loss coefficient. Similar values of $\alpha$, in the range $5-10 \mathrm{~cm}^{-1}$, are obtained by our two analysis techniques. In the case of biosilica waveguides deposited on quartz substrates, values of $\alpha$ in the range $15-20 \mathrm{~cm}^{-1}$ are measured. Finally, different loss coefficients are measured by aligning the polarization of the incident laser parallel to the $y$ axis $\left(\alpha_{/ /}\right)$or to the $x$ axis $\left(\alpha_{\perp}\right)$ in the cross-sectional plane of the fibre finding a $\alpha_{/ /} / \alpha_{\perp}$ ratio of $\cong 1.5$.

\section{Discussion}

In the present work, biosilica microstructures are obtained by exploiting a soft lithographic approach and the enzymatic reaction of the recombinant protein silicatein- $\alpha$, belonging to the cathepsin proteases family. While the other cathepsin proteases are characterized by their catalytic triad Cys, His and Asp, the triad in the silicateins comprises, instead of Cys, a Ser residue ${ }^{23-28}$. One or two Ser-rich cluster(s) are implicated in the binding of the silicateins to the polymeric silicate ${ }^{34}$ and are located in the segment with the highest sequence similarity (signature) between the demosponge and hexactinellid silicateins, from $\mathrm{aa}_{292}$ to $\mathrm{aa}_{300}{ }^{24,35,36}$. Aiming to produce a functionally-active recombinant silicatein- $\alpha$ protein, first a structural model is predicted using the known crystal structure of cathsilicatein, a chimeric mutant of the human cathepsin $\mathrm{L}^{29}$. The structure prediction study confirms our recombinant silicatein to have a correct structure for allowing biosilicification reactions: monomeric silica precursors, such as TEOS (Fig. 1), can be docked in the active centre of silicatein molecules and catalyzed for the silica polycondensation. This result, in agreement with previous experimental results showing this enzymatic activity well preserved even after successful immobilization on different substrates ${ }^{9,37,38}$, states the possibility to use recombinant silicatein- $\alpha$ instead of the full protein, i.e. without sacrificing marine or freshwater living organisms. In the natural formation of glass spicules in sponges, the structure of silica fibres is composed by an organic filamentous core, mainly made of proteins, that promotes the polycondensation of silica encouraging the deposition of lamellar silica layers with progressively decreasing organic content upon moving from the central core to the spicule 
a

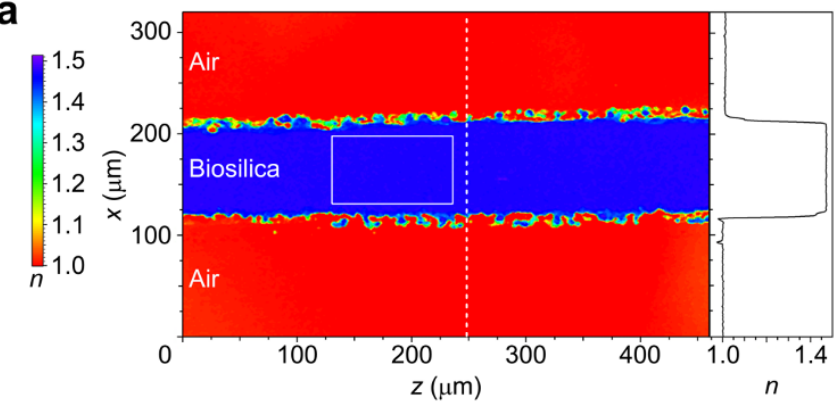

b

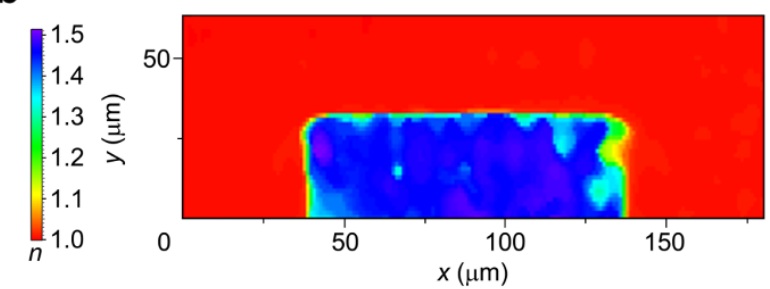

C

d Light Intensity (a.u.)
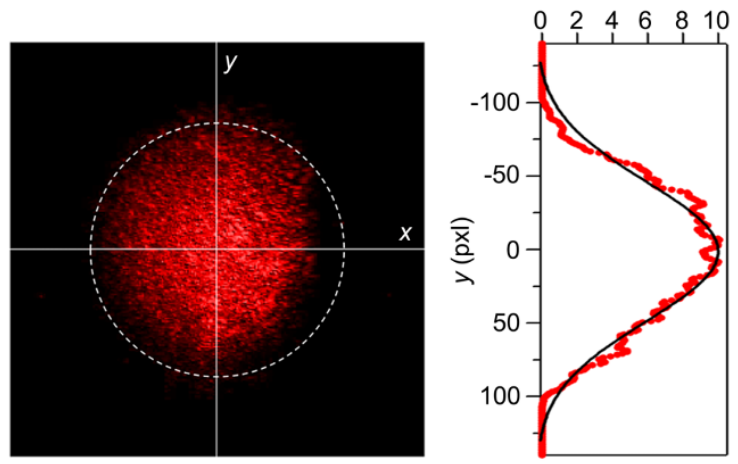

e

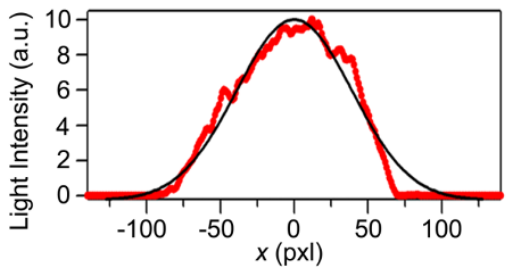

Figure 3 Biosilica waveguides and coupling of light to free space. Top (a) and cross-sectional (b) refractive index maps of a biosilica wire with width $100 \mu \mathrm{m}$, deposited onto a borosilicate glass substrate

$(n=1.534 \pm 0.002$ at $\lambda=405 \mathrm{~nm}$, thickness $160 \mu \mathrm{m}$ ). In (a) we also show an index profile collected along the vertical dotted line. Mapping obtained by confocal imaging of the light back-reflected from the substrate/biosilica or air/biosilica interface (see Supplementary Information for details).

(c) Multi-mode light spot $(\lambda=633 \mathrm{~nm})$ transmitted by a fibre. A laser is coupled into the fibre, and transmitted photons are collected through an objective lens (N.A. 0.10) and imaged by a CCD. (d-e) Light intensity profiles (red dots), measured along the vertical $(y)$ and horizontal $(x)$ cross section lines in (d) and (e), respectively, with corresponding best fits by Gaussian curves (superimposed lines).

periphery ${ }^{19,26}$. Aiming to mimic this mechanism, silicatein- $\alpha$ is first patterned by microfluidics on a hydroxylated $\mathrm{Si} / \mathrm{SiO}_{2}$ substrate, and then incubated in a silica precursor solution. After patterning, the fractal dimension of the resulting fibrillar structure $(\approx 1.7)$ is found identical to those reported for networks of proteins isolated by $T$. aurantia spicules ${ }^{30}$, which propose these fabricated microstructures as promising candidates for functionally directing biosilicification. The presence of silicon after incubation in TEOS solution, confirmed by elemental analysis, suggests that the silica polycondensation reaction occurs.
Table 1 | Measured refractive index for achieved biosilica. Data are obtained by confocal imaging of light reflected from borosilicate/biosilica interfaces (see the text). The value of $n$ at $633 \mathrm{~nm}$ is extrapolated by the fit of experimental data through a Cauchy model with coefficients ( \pm s.d.) $A=1.46 \pm 0.01$ and $B=(3.5 \pm$ 1.5) $\times 10^{3} \mathrm{~nm}^{2}$

\begin{tabular}{lc}
$\lambda(\mathrm{nm})$ & $n( \pm$ s.d.) \\
\hline 405 & $1.482 \pm 0.003$ \\
457.9 & $1.480 \pm 0.003$ \\
488.0 & $1.478 \pm 0.003$ \\
514.5 & $1.472 \pm 0.003$ \\
633.0 & 1.47 \\
\hline
\end{tabular}

By studying the optical properties of these biomineralised microstructures, an enhancement of the spatially-averaged $n$ value is found after the biosilicification process, thus well mimicking the biosilicification process observed in sponge spicules in-vivo ${ }^{19}$. Both the refractive index and the wavelength dispersion are very close to typical values and behaviour of amorphous quartz $(n \cong 1.46$ at $\lambda=633 \mathrm{~nm}$, Table 1$)^{39}$. Moreover, we report the first quantitative assessment of the waveguiding performances of biosilicificated microstructures, given the previous lack of such data even on natural biosilica. The optical losses from our biosilica microstructures can be induced, besides absorption from the biosilica or protein bulk materials, by photon scattering from the micro- and nanoscale discontinuities and defects of the fibre surface, as displayed by the micrograph in the inset of Fig. 4a. We find that free-standing samples exhibit lower values of $\alpha$ than biosilica waveguides deposited on quartz substrates $\left(\alpha=5-10 \mathrm{~cm}^{-1}\right.$ vs. $15-20 \mathrm{~cm}^{-1}$, respectively). This is physically expected, because of the partial coupling of the guided photons into underlying quartz, whose refractive index $(n$ $\cong 1.46$ ) is very close to that of the fibre material, thus disfavouring light confinement within biosilica. Overall, these optical losses are still almost an order of magnitude higher with respect to the stateof-the-art fused silica and polymer waveguides used for on-chip applications (see Table S1 in the Supplementary Information). Nevertheless, these results could open the way to a versatile and biocompatible approach towards the integration of optical elements in lab on chips ${ }^{22,40}$. Strategies to decrease scattering losses include a better control of the microfluidic process to prevent protein sticking on PDMS and obtain smoother walls, and the use of a cladding layer with a refractive index close to that of biosilica, thus decreasing the index contrast at the silica surface $e^{41,42}$.

By aligning the polarization of the incident laser parallel to the $y$ axis $\left(\alpha_{/ /}\right)$or to the $x$ axis $\left(\alpha_{\perp}\right)$ in the cross-sectional plane of the fibre, we find a $\alpha_{/ /} / \alpha_{\perp}$ ratio of $\cong 1.5$, suggesting a spatial anisotropy within the microstructure. In fact, SEM images of the cross section of the biosilica fibres (Fig. 4b) reveals the presence of sub-micron, ordered arrays. These supramolecular structures, providing a cross-sectional projection of the elongated features observed on the surface of the fibres (Fig. 4c), are likely induced by the assembly and orientation of protein filaments within bundles along the flow direction of microfluidics ${ }^{43}$. Several optical properties, such as dichroism and birefringence, can be associated to the spatial anisotropy of such bundles of filaments, and will deserve further investigation. These features, including the observed dependence of losses on the polarization of the transmitted radiation, can result from isotropic materials arranged into an anisotropic structure, whose typical length-scale (sub- $\mu \mathrm{m})$ is intermediate between the size of the molecular constituents and the light wavelength, as found here and for some crystalline materials at molecular level or self-assembled ordered structures from nanoparticles ${ }^{44}$.

In summary, we have developed a straightforward approach to realize microstructures by biosilicification in-vitro, assessing the 
a

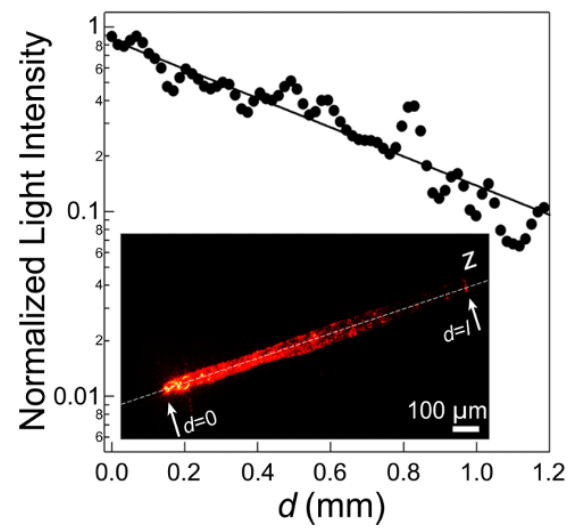

b

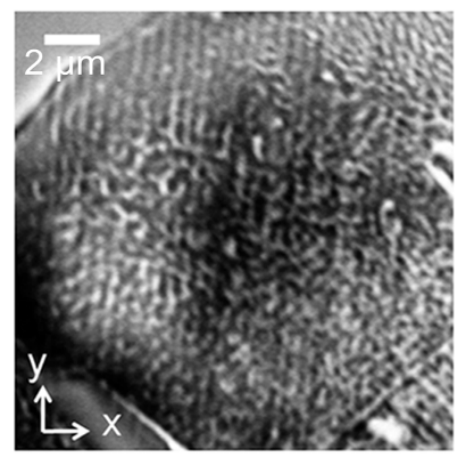

C

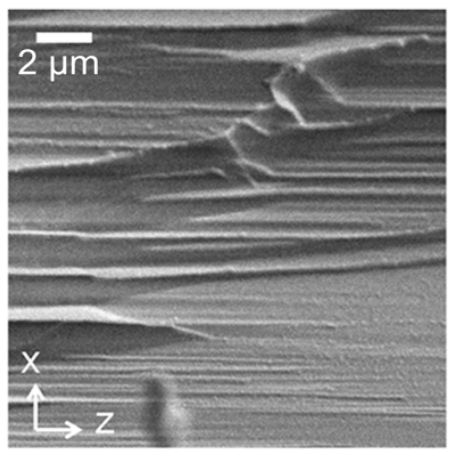

Figure $4 \mid$ Waveguiding properties of biosilica microstructures. (a) Dependence of the intensity, $I$, of the guided light inside a free-standing biosilicificated fibre on the distance, $d$, of the scattering points on the fibre surface from the entrance tip. The superimposed continuous line is the best fit of the data to the equation $I=I_{0} \exp (-\alpha d) . \lambda=633 \mathrm{~nm}$. Inset: micrograph of a biosilica fibre coupled to He-Ne laser beam. The arrows indicate the fibre tips. The light is coupled into the fibre at $d=0$ and exits from the opposite tip at $d=l$, where $l$ is the fibre length. (b-c) SEM micrographs of the fibre cross section (b) and body (c). Bars $=2 \mu \mathrm{m}$. The coordinates, $x, y$ and $z$ are shown to better clarify the orientation of the imaged surfaces. The two SEM images evidence the section and the external surface of a bundle of filamentous structures along the longitudinal fibre axis $(z)$, respectively.

achievable optical properties. The promised benefits are given by using low temperature and biologic systems, without sacrificing marine or freshwater living organisms. Achieving on-demand geometries of increasing complexity, is also possible by soft lithography patterning applied to biosilicification, and represents a promising goal for future applications.

\section{Methods}

Recombinant silicatein production. The silicatein- $\alpha$ cDNA from $S$. domuncula is introduced into an oligo-His expression vector (pQE30, Qiagen, Hilden, Germany), used for transforming the E. coli host strain M15 (Qiagen) ${ }^{9}$. A BIOSTAT Aplus bioreactor (Sartorius Stedim Biotech, Aubagne, France) is used for the bacterial culture. Isopropyl $\beta$-D-thiogalactopyranoside is employed for inducing the expression of the fusion protein. Finally, the protein is collected, purified by affinity chromatography and refolded.

Microfluidics and biomineralisation. Masters for defining the microfluidic channels and protein patterning are fabricated by photolithography, and then replicated by moulding into an elastomer. Pressure-driven fluidics is then performed with microfluidic devices, assembled by the textured elastomeric element. Silicatein solution with a concentration of $0.7 \mathrm{mg} / \mathrm{mL}$ is injected into the channels by a syringe pump. After peeling off, silicatein microstructures stay on the substrate with a yield of $90 \%$. Some silicatein fibres $(10 \%)$ are physically trapped in the recessed features of the PDMS mould and once released can be handled as free standing fibres. These are finally incubated in a silica precursor solution (TEOS, 99\% pure) for $120 \mathrm{~h}$ to realize biosilica fibres (see Supplementary Information for details).

Biomineralised microstructure characterization. Obtained fibres are investigated by SEM, and their composition is estimated by energy dispersive $\mathrm{x}$-ray spectroscopy (EDS). The refractive index of silicatein and biosilica surfaces are obtained by confocal imaging of the light back-reflected from a borosilicate glass/silicatein, biosilica/air, and borosilicate/biosilica interfaces. Propagation loss measurements are performed by measuring the intensity of light $(\lambda=633 \mathrm{~nm})$ scattered from the fibre surface, being directly proportional to the light intensity propagating along the fibre axis. The optical losses are also measured by means of the light exiting the tip of the fibre, providing the ratio of the power of the light exiting the tip $\left(P_{\text {out }}\right)$ to the power of the beam laser coupled into the fibre $\left(P_{i n}\right)$. The two used techniques provide same values of waveguide losses.

1. Bäuerlein, E., Behrens, P. \& Epple, M. Handbook of Biomineralization (Wiley$\mathrm{VCH}, 2007)$.

2. Estroff, L. A. Introduction: Biomineralization. Chem. Rev. 108, 4329-4331 (2008).

3. Erlich, H. Biological materials of marine origin-Invertebrates (Springer Verlag, 2010).

4. Dickerson, M. B., Sandhage, K. H. \& Naik, R. R. Protein- and peptide-directed syntheses of inorganic materials. Chem. Rev. 108, 4935-4978 (2008).

5. Kröger, N. \& Sandhage, K. H. From diatom biomolecules to bioinspired syntheses of silica- and titania-based materials. MRS Bulletin 35, 122-126 (2010).
6. Sarikaya, M., Tamerler, C., Jen, A. K. Y., Shulten, K. \& Baneyx, F. Molecular biomimetics: nanotechnology through biology. Nature Materials 2, 577-585 (2003).

7. Thomas, A., Schlaad, H., Smarsly, B. \& Antonietti, M. Replication of lyotropic block copolymer mesophases into porous silica by nanocasting: Learning about finer details of polymer self-assembly. Langmuir 19, 4455-4459 (2003).

8. Belcher, A. M., Wu, X. H., Christensen, R. J., Hansma, P. K., Stucky, G. D. \& Morse, D. E. Control of crystal phase switching and orientation by soluble mollusk-shell proteins. Nature 381, 56-58 (1996).

9. Polini, A. et al. Biosilica electrically-insulating layers by soft lithography-assisted biomineralisation with recombinant silicatein. Adv. Mater. 23, 4674-4678 (2011).

10. Politi, A., Cryan, M. J., Rarity, J. G., Yu, S. Y. \& O’Brien, J. L. Silica-on-Silicon waveguide quantum circuits. Science 320, 646-649 (2008).

11. Ferrera, M. et al. Low-power continuous-wave nonlinear optics in doped silica glass integrated waveguide structures. Nat. Photon. 2, 737-740 (2008).

12. Smith, C. M. et al. Low-loss hollow-core silica/air photonic bandgap fibre. Nature 424, 657-659 (2003).

13. Büchel, K. H., Moretto, H. H. \& Woditsch, P. Industrial Inorganic Chemistry (Wiley-VCH, 2000).

14. Doering, R. \& Nishi, Y. Handbook of Semiconductor Manufacturing Technology (Taylor \& Francis Group, 2007).

15. Iler, R. K. The Chemistry of Silica: Solubility, Polymerization, Colloid and Surface Properties, and Biochemistry (Wiley-VCH, 1979).

16. Ehrlich, H. Silica biomineralization in sponges. in Encyclopedia of Geobiology Reitner, J. \& Thiel, V. (Springer Verlag, 2011).

17. Cattaneo Vietti, T. et al. Optical fibres in an Antarctic sponge. Nature 383, 397-398 (1996).

18. Müller, W. E. G. et al. Novel photoreception system in sponges? Unique transmission properties of the stalk spicules from the hexactinellid Hyalonema sieboldi. Biosens. Bioelectron. 21, 1149-1155 (2006).

19. Aizenberg, J., Sundar, V. V., Yablon, A. D., Weaver, J. C. \& Chen, G. Biological glass fibres: Correlation between optical and structural properties. Proc. Natl. Acad. Sci. USA 101, 3358-3363 (2004).

20. Kul'chin, Y. N. et al. Optical fibres based on natural biological minerals - sea sponge spicules. Quantum Electron. 38, 51-55 (2008).

21. Osellame, R., Maselli, V., Martinez Vazquez, R., Ramponi, R. \& Cerullo, G. Integration of optical waveguides and microfluidic channels both fabricated by femtosecond laser irradiation. Appl. Phys. Lett. 90, 231118-231120 (2007).

22. Lee, K. S., Lee, H. L. T. \& Ram, R. J. Polymer waveguide backplanes for optical sensor interfaces in microfluidics. Lab Chip 7, 1539-1547 (2007).

23. Shimizu, K., Cha, J., Stucky, G. D. \& Morse, D. E. Silicatein $\alpha$ : Cathepsin L-like protein in sponge biosilica. Proc. Natl. Acad. Sci. USA 95, 6234-6238 (1998).

24. Cha, J. N. et al. Silicatein filaments and subunits from a marine sponge direct the polymerization of silica and silicones in vitro. Proc. Natl. Acad. Sci. USA 96, 361-365 (1999).

25. Krasko, A. et al. Cathepsin, a major protease of the marine sponge Geodia cydonium: purification of the enzyme and molecular cloning of cDNA. Mol. Mar. Biol. Biotechnol. 6, 296-307 (1997).

26. Müller, W. E. G. et al. Formation of siliceous spicules in the marine demosponge Suberites domuncula. Cell. Tissue Res. 321, 285-297 (2005).

27. Krasko, A. et al. Expression of silicatein and collagen genes in the marine sponge Suberites domuncula is controlled by silicate and myotrophin. Eur. J. Biochem. 267, 4878-4887 (2000). 
28. Müller, W. E. G. et al. Poly(silicate)-metabolizing silicatein in siliceous spicules and silicasomes of demosponges comprises dual enzymatic activities (silicapolymerase and silica-esterase). FEBS J 275, 362-370 (2008).

29. Fairhead, M. et al. Crystal structure and silica condensing activities of silicatein $\alpha$ cathepsin L chimeras. Chem. Commun. 1765-1767 (2008).

30. Murr, M. M. \& Morse, D. E. Fractal intermediates in the self-assembly of silicatein filaments. Proc. Natl. Acad. Sci. USA 102, 11657-11662 (2005).

31. Ehrlich, H. et al. Mineralization of the metre-long biosilica structures of glass sponges is templated on hydroxylated collagen. Nature Chemistry 2, 1084-1088 (2010)

32. Born, M. \& Wolf, E. Principles of Optics: Electromagnetic Theory of Propagation, Interference and Diffraction of Light (Cambridge University Press, 1964).

33. Olsen, C. M., Trewhella, J. M., Fan, B. \& Oprysko, M. M. Propagation properties in short lengths of rectangular epoxy waveguides. IEEE Photonic. Tech. L. 4, 145-148 (1992).

34. Lobel, K. D., West, J. K. \& Hench, L. L. Computational model for protein-mediated biomineralization of the diatom frustule. Mar. Biol. 126, 353-360 (1996).

35. Müller, W. E. G. et al. Silicatein expression in the hexactinellid Crateromorpho meyeri: the lead marker gene restricted to siliceous sponges. Cell. Tissue Res. 333, 339-351 (2008).

36. Müller, W. E. G. et al. Siliceous spicules in marine demosponges (example Suberites domuncula). Micron 37, 107-120 (2006).

37. Tahir, M. N. et al. Monitoring the formation of biosilica catalysed by histidintagged silicatein. Chem. Comm. 24, 2848-2849 (2004)

38. Rai, A. \& Perry, C. C. Facile fabrication of uniform silica films with tunable physical properties using silicatein protein from sponges. Langmuir 26, 4152-4159 (2010)

39. Malitson, I. H. Interspecimen comparison of the refractive index of fused silica. J. Opt. Soc. Am. 55, 1205-1209 (1965).

40. Mogensen, K. B., El-Ali, J., Wolff, A. \& Kutter, J. P. Integration of polymer waveguides for optical detection in microfabricated chemical analysis systems. Appl. Opt. 42, 4072-4079 (2003).

41. Lee, K. K., Lim, D. R., Kimerling, L. C., Shin, J. \& Cerrina F. Fabrication of ultralow-loss $\mathrm{Si} / \mathrm{SiO}_{2}$ waveguides by roughness reduction. Opt. Lett. 26, 1888-1890 (2001).
42. Suzuki, S., Yanagisawa, M., Hibino, Y. \& Oda, K. High-density integrated planar lightwave circuits using $\mathrm{SiO}_{2} \mathrm{GeO}_{2}$ waveguides with a high refractive index difference. J. Lightwave Technol. 12, 790-796 (1994).

43. Lee, P., Lin, R., Moon, J. \& Lee, L. P. Microfluidic alignment of collagen fibres for in vitro cell culture. Biomed. Microdevices 8, 35-41 (2006).

44. Talapin, D. V. et al. CdSe and CdSe/CdS nanorod solids. J. Am. Chem. Soc. 126, 12984-12988 (2004).

\section{Acknowledgements}

This work is financially supported by the BIO-LITHO European project $\left(6^{\text {th }}\right.$ Framework Program, NMP). W.E.G. Müller is holder of an ERC Advanced Research Grant. The authors thank G. Potente for SEM, M. Polo for help in the propagation loss measurements, and L. Martiradonna for EDS measurements.

\section{Author contributions}

H.C.S., W.E.G.M. and D.P. designed the research. X.W., H.C.S. and W.E.G.M. performed and analyzed the biological experiments. A.P. and S.P. performed lithographies. A.P., S.P. and A.C. collected and analyzed optical and structural data. A.P., S.P., R.C., A.C. and D.P. discussed waveguiding data. A.P., W.E.G.M. and D.P. wrote the paper. All authors discussed the results and commented on the manuscript.

\section{Additional information}

Supplementary information accompanies this paper at http://www.nature.com/ scientificreports

Competing financial interests: The authors declare no competing financial interests.

License: This work is licensed under a Creative Commons

Attribution-NonCommercial-ShareAlike 3.0 Unported License. To view a copy of this license, visit http://creativecommons.org/licenses/by-nc-sa/3.0/

How to cite this article: Polini, A. et al. Optical properties of in-vitro biomineralised silica. Sci. Rep. 2, 607; DOI:10.1038/srep00607 (2012). 